\title{
The Sustained Rotavirus Vaccination Impact on Nosocomial Infection, Duration of Hospital Stay, and Age: The RotaBIS Study (2005-2012)
}

Baudouin Standaert · Danielle Strens $\cdot$ Xiao Li ·

Nadia Schecroun · Marc Raes

Received: June 20, 2016/Published online: October 6, 2016

(C) GSK group of companies, D. Strens and M. Raes 2016. This article is published with open access at Springerlink.com

\section{ABSTRACT}

Introduction: The benefits of rotavirus (RV) vaccination in developed countries have focused on reductions in mortality, hospitalization and medical visits, and herd protection. We investigated other aspects related to RV-induced nosocomial infection, duration of hospital stay, age shift, and sustained vaccine impact (VI) over time.

Method: RotaBIS (Rotavirus Belgian Impact Study; ClinicalTrials.gov identifier, NCT01563146) annually collects retrospective data on hospitalization linked to RV testing in children up to 5 years old from 11 pediatric

Enhanced content To view enhanced content for this article go to http://www.medengine.com/Redeem/ 60E6F0603A3DF39E.

B. Standaert $(\varangle) \cdot X$. Li

GSK Vaccines, Wavre, Belgium

e-mail: baudouin.a.standaert@gsk.com

D. Strens

Realidad, Grimbergen, Belgium

N. Schecroun

Keyrus Biopharma (c/o GSK Vaccines, Wavre,

Belgium), Lasne, Belgium

M. Raes

Pediatrics, Jessa Hospital, Hasselt, Belgium wards located all over Belgium. Data from 2005 to 2012 have been split in pre- (2005-2006) and post-vaccination (2007-2012) period. Information was collected on age, gender, RV test result, nosocomial infection caused by RV and duration of hospital stay.

Results: Over the 6-year period after the introduction of the RV vaccine, an $85 \%$ reduction in nosocomial infections was observed (221 in 2005 to 33 in 2012, $p<0.001$ ). A significant reduction of almost 2 days in average duration of hospital stay per event was observed overall (7.62 days in 2005 to 5.77 days in 2012, $p<0.001)$. The difference is mainly explained by the higher reduction in number of nosocomial infections. A pronounced age shift $(+24 \%$, $p<0.01)$ of RV nosocomial infection to infants $\leq 2$ months old was observed, increasing with length of post-vaccination period. VI was maintained over the follow-up $( \pm 79 \%$ VI per birth cohort). A decrease was seen depending on age, 85\% (95\% CI 76-91\%) in the youngest to 63\% (95\% CI 22-92\%) in the oldest age group.

Conclusion: The higher reduction in nosocomial infection may affect the overall average duration of hospital stay for RV infection. No change in VI by birth cohort, 
but a reduction by age group was observed. These findings could be important for decision-makers considering the introduction of universal mass RV vaccination programs.

Trial registration: ClinicalTrials.gov identifier, NCT01563146.

Funding: GlaxoSmithKline Biologicals SA (Rixensart, Belgium).

Keywords: Age shift; Bed occupancy; Duration of hospital stay; Hospitalization; Nosocomial infection; Quality of care; Rotavirus; Vaccination; Vaccine impact

\section{INTRODUCTION}

Rotavirus (RV) infection is a well-known cause of acute gastro-enteritis leading to severe diarrhea in small children. It shows a seasonal pattern in the more temperate climates, resulting in epidemic peaks during winter and spring $[1,2]$. As a result pediatric wards are often overwhelmed with a high bed occupancy rate due to diarrheal disease [3]. High rates of infant hospitalization for other conditions that occur in the same period, such as bronchiolitis, can further compound the problem of overcrowding. [4]. The use of vaccines could help to improve management of this recurrent annual health care problem [5]. Different vaccines have demonstrated a high efficacy against severe disease in infants and young children. Two vaccines are currently on the market at the global level, a two-dose vaccine Rotarix ${ }^{\mathrm{TM}}$ (GSK, Rixensart, Belgium) [6, 7] and a three-dose vaccine Rotateq ${ }^{\mathrm{TM}}$ (Merck and Co. Inc, Whitehouse Station, New Jersey, United States) [8]. Belgium was one of the first countries in Europe to introduce the RV vaccine with a co-payment scheme [9]. The vaccines have been reimbursed since November 2006 (first Rotarix and subsequently Rotateq, 1 year later) [10]. A high vaccine uptake (above $85 \%$ ) in the first year of introduction of $\mathrm{RV}$ vaccination has been maintained over the years $[9,11]$. To date, the vaccination program has had more than 7 years' impact on the local health care system.

In 2007, we initiated the RotaBIS (Rotavirus Belgian Impact Study) study. This project annually collects retrospective data for the past year on RV testing linked to the hospitalization of children aged under 5 years in 11 hospitals located all over Belgium [9]. Based on RotaBIS, we reported last year on the general effect the vaccines have had on hospitalization, expressing the number of positive test results for RV by age and year and comparing the pre- versus the post-vaccination periods [12]. The results demonstrated sustained vaccine-associated protection over time, i.e., a reduction of RV-related hospitalizations observed after 7 years of around $75 \%$, with a high herd protection level of $26.5 \%$ in the first year of the vaccine introduction. In addition, data on many other parameters were systematically collected during the whole follow-up period of the RotaBIS project. These additional data are now analyzed in the present study to address further questions on the impact of the vaccine on RV-induced nosocomial infections [13], the average duration of hospital stay being for a certain period a surrogate marker for disease severity [14], a potential more pronounced age shift of $\mathrm{RV}$ disease after the introduction of the vaccine [15], and the sustainability of the vaccine impact (VI) over time [12].

Based on the data available since 2005, we have attempted to investigate whether the total value of the RV vaccine extends beyond the reduction in hospitalization rate and the generation of a high level of herd protection in the early period of the vaccine introduction. 
Information on these additional questions could help to test earlier conclusions that RV vaccination could improve quality of care (QoC) in the hospital environment, as previously suggested [16].

\section{METHODS}

\section{Data Source}

The RotaBIS is a retrospective hospital database study (ClinicalTrials.gov identifier, NCT01563146) previously published [9]. The study annually collects retrospective data on hospitalization linked to RV testing in children aged up to 5 years from 11 pediatric wards located all over Belgium, which represent around 450 pediatric beds (16\% of the total available in the country). Data from 2005 to 2012 have been split in pre- (2005-2006) and post-vaccination (2007-2012) period (reimbursement of the vaccine was introduced in November 2006) [17].

The information assembled was the following: center code, age, gender, date of sampling, RV test results, date of hospital admission and discharge, clinical outcome (ambulatory or hospitalized), and evidence of nosocomial infection. An RV infection was identified as nosocomial if the stool sample tested was RV positive $48 \mathrm{~h}$ or more after the day of non-diarrhea-related hospital admission. The duration of nosocomial infection was reported as the full period of hospitalization and the period from which the rotavirus test was positive. No information on the RV vaccination status of the child was available. The data were anonymized before any analysis occurred. All procedures followed were in accordance with the ethical standards of the responsible committee in each center. Approval for the study was obtained from each center each year the data were collected.

\section{Data Analysis}

Among the RV tests performed in the children admitted in these hospitals during the evaluation period, the proportion of RV-positive tests was calculated for each participating center, per month and per year, for six different age groups: 0-2 months, >2-12 months, >1-2 years, $>2-3$ years, $>3-4$ years, and $>4-5$ years. Data among the sub-group of children aged $\leq 2$ months were also analyzed because, even if they were too young for vaccination, they could experience a herd protection effect after the vaccine was introduced.

The data were analyzed per year (2005-2012) for the number of RV-induced nosocomial infections during the peak periods of January until the end of May for the six age groups. The data were also compared per year relative to their distribution by age group: pre-vaccination versus post-vaccination (first year, second year, $n$th year, ..., sixth year post-vaccination). The average duration of hospital stay for the treatment of RV diarrhea per year and per age group is reported. The cumulative proportion of $\mathrm{RV}$ patients as a function of the duration of their hospital stay per year is also evaluated. It allows the identification of the proportion of patients with a very long hospital stay. These patients should be considered separately, as their long stay is more influenced by other diseases for which they were hospitalized than by the rotavirus infection they acquired during their stay. The hypothesis that the introduction of the vaccine may have reduced the overall average duration per rotavirus disease event and per source of infection (nosocomial and community-acquired) has been tested. The 
duration of the RV-induced nosocomial infection events were split into a pre- and a post-sample period. Finally, the analysis estimated the impact of vaccination on the overall bed occupancy during the peak period by source of infection (community-acquired and nosocomial) for the year 2005 (pre-vaccination) and 2012 (post-vaccination).

The VI is reported over time on RV-induced hospitalizations by age group (i) and birth cohort (y). A full definition for calculating the VI has been presented in the previous article on the RotaBIS study [12], but here the relative value is measured by calculating the ratio of hospitalization rate post-vaccination (Hosp $\mathrm{p}_{\text {viy }}$ ) to the hospitalization rate for the same age groups pre-vaccination for one reference year,

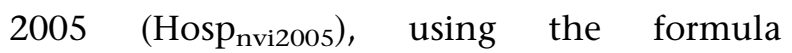
$\left(\mathrm{VI}=\mathrm{Hosp}_{\text {viy }} / \mathrm{Hosp}_{\text {nvi2005 }}-1\right)$. We only investigated the direct VI and did not consider age groups that are under the influence of herd protection. This analysis should indicate whether the VI is maintained over time by year and age.

To measure the relative importance of the disease as a function of age that may shift over time after vaccine introduction, we calculated the proportional distribution of the disease over the different age groups in each year. We then plotted on a graph the proportional results per age group in each year and observed shifts in those proportions.

\section{Assumptions}

A few assumptions underlie the comparison of the annual number of RV-positive tests. One assumption is that the catchment area of each of the participating centers remained the same across the whole observation period of 8 years. Another assumption is that no change occurred during the period in the disease management behavior of testing children up to 5 years old for RV disease. This means that if fewer tests were performed after the introduction of the vaccine, this is mainly due to fewer suspected cases presenting to the hospital unit and not to a change in behavior of physicians who may have decided to perform RV tests less frequently after vaccine introduction. Therefore, the most relevant comparison between the years is the accumulated number of RV-positive tests and not the proportion of RV-positive tests per year. A third assumption is that during the whole observation period of 8 years there was no change in the criteria for hospitalizing patients with rotavirus disease, given that hospital beds were becoming unoccupied during the peak period after the vaccine had been introduced. Reduced bed occupancy could become an incentive for trying to get those beds occupied by changing the admission criteria.

\section{Statistics}

Results were tested for statistical significance using Chi-square tests for the trend of proportional and absolute data over the years. Non-parametric rank testing (Kruskal-Wallis test and Mann-Whitney test) or ANOVA testing after a normality check of the data distribution was used for comparing the values. If not normally distributed, log-norm transformation was applied. 95\% confidence intervals (CI) were measured for VI values using the Wilson method [18]. The statistical tests were performed with GraphPad Prism 6.2 for Windows, GraphPad Software, La Jolla California USA, www.graphpad.com. The cutoff value for significance was $p=0.05$. 


\section{RESULTS}

\section{RV-induced Nosocomial Infections}

Figure 1 and Table 1 present the results for $\mathrm{RV}$-induced nosocomial infection by year. There is a decrease observed in RV-induced nosocomial infections from $2005(n=221)$ to $2012(n=33)$ (Chi-square test for trend: 10.96; $p<0.001$ ), with a relative reduction of $85 \%$ when comparing the two extreme years. The relative contribution of nosocomial $\mathrm{RV}$ infections to the total number of $\mathrm{RV}$ hospitalizations also dropped from $17 \%$ in 2005 to $10 \%$ in 2012 (Table 1) (Chi-square for relative trend $=11.13, p<0.001)$. The peak observed in April 2011 in Fig. 1 reflects an unusually high number of cases from a single center during that month $(8 / 21$ cases, $38 \%$ of the total).

\section{Hospital Bed Occupancy and Length of Stay}

The above data indicated a dramatic reduction in the number of nosocomial infections after the introduction of the vaccine. We investigated the contribution of rotavirus to bed days occupied during the peak period of rotavirus disease pre-vaccination and a few years after the vaccine was introduced. Table 2 summarizes the collected numbers for 2005 pre-vaccination and 2012 post-vaccination (there were statistically significant differences

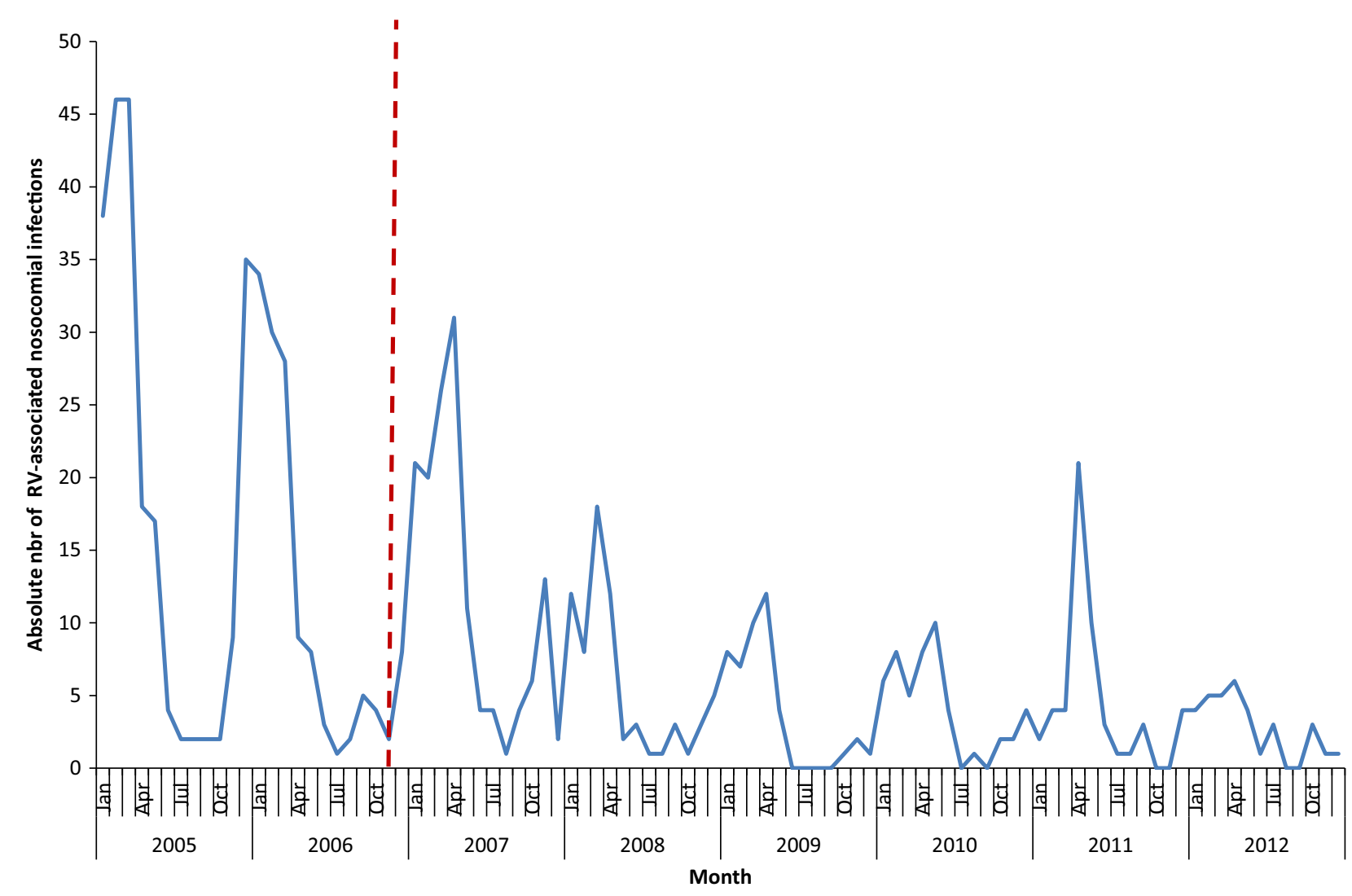

Fig. 1 Spread of RV-induced nosocomial infections over the years. Dotted red line indicates when RV vaccine was reimbursed. $R V$ rotavirus 
Table 1 The relative contribution of RV-induced nosocomial infections to positive RV test results by year

\begin{tabular}{lllll}
\hline Year & $\begin{array}{l}\text { RV-induced } \\
\text { non-nosocomial } \\
\text { infections }\end{array}$ & $\begin{array}{l}\text { RV-induced } \\
\text { nosocomial } \\
\text { infections }\end{array}$ & Total & $\begin{array}{l}\text { \% RV-induced } \\
\text { nosocomial } \\
\text { infections (\%) }\end{array}$ \\
\hline 2005 & 1103 & 221 & 1324 & 17 \\
2006 & 1265 & 134 & 1399 & 10 \\
2007 & 694 & 143 & 837 & 17 \\
2008 & 462 & 69 & 531 & 13 \\
2009 & 318 & 45 & 363 & 12 \\
2010 & 336 & 50 & 386 & 13 \\
2011 & 359 & 53 & 412 & 13 \\
2012 & 294 & 33 & 327 & 10 \\
\hline
\end{tabular}

$R V$ rotavirus

Table 2 Bed-days occupied pre- (2005) and post-vaccination (2012) during the peak-period overall and by source of infection (community-acquired and nosocomial)

\begin{tabular}{lllcrr}
\hline Year & Month & $\begin{array}{l}\text { Total bed days } \\
\text { available }\end{array}$ & $\begin{array}{c}\text { Overall rotavirus } \\
\text { occupancy (\%) }\end{array}$ & $\begin{array}{c}\text { Community- } \\
\text { acquired (\%) }\end{array}$ & \multicolumn{1}{c}{$\begin{array}{l}\text { Nosocomial } \\
\text { (\%) }\end{array}$} \\
\hline 2005 & 1 & 13,640 & $930(6.8 \%)$ & $541(4.0 \%)$ & $389(2.9 \%)$ \\
& 2 & 12,320 & $1433(11.6 \%)$ & $1101(8.9 \%)$ & $322(2.7 \%)$ \\
& 3 & $1649(12.1 \%)$ & $1288(9.4 \%)$ & $361(2.6 \%)$ \\
& 4 & $543(4.1 \%)$ & $449(3.4 \%)$ & $94(0.7 \%)$ \\
& 5 & 13,640 & $280(2.1 \%)$ & $217(1.6 \%)$ & $63(0.5 \%)$ \\
& 13,200 & $4835(7.3 \%)$ & $3596(5.4 \%)$ & $1239(1.9 \%)$ \\
& Total & 66,440 & $113(0.8 \%)$ & $57(0.4 \%)$ & $56(0.4 \%)$ \\
& 1 & 13,981 & $137(1.1 \%)$ & $97(0.8 \%)$ & $40(0.3 \%)$ \\
& 2 & 12,628 & $145(1.0 \%)$ & $113(0.8 \%)$ & $32(0.2 \%)$ \\
& 3 & 13,981 & $288(2.1 \%)$ & $262(1.9 \%)$ & $26(0.2 \%)$ \\
& 4 & 13,530 & $168(1.2 \%)$ & $145(1.0 \%)$ & $23(0.2 \%)$ \\
& 5 & $851(1.2 \%)$ & $674(1.0 \%)$ & $177(0.3 \%)$ \\
\hline
\end{tabular}

in the reduced proportions of 2005 compared with 2012, Chi-square, $d f=3017, p<0.001$ ). The denominator is different for the two years, as 11 more pediatric beds were available in 2012. The contribution of rotavirus disease to the overall bed occupancy varied per month during the pre-vaccination period, with peaks up to $12 \%$ of the pediatric beds. Nosocomial infections could have a peak contribution that occurs independently of the community-acquired peak. The impact of the vaccine on bed occupancy is that 6 years after 
its introduction, close to 3984 bed days, or $6 \%$, are available during the peak period per year, which can be used for purposes other than rotavirus disease.

\section{Overall Average Duration}

The overall average duration of a hospital stay for an RV-induced diarrhea event also decreased from 7.62 to 5.77 days, which is close to a 2-day average gain per event, (ANOVA testing after log-normal transformation, $F(7,4389)=7.706$, $p<0.001$ ) (see Fig. 2). During the 5 most recent years, a clear trend reduction in average duration was observed, with the standard errors of the mean also becoming smaller (ANOVA testing after log-normal transformation, $F(4,1544)=8.599, p<0.001)$.

The spread of hospital duration was heavily skewed to the right (long tail to the right) pre-vaccination in 2005, as shown in Fig. 3. After the introduction of the vaccine this skewness to the right became much less pronounced, as seen for the year 2012.

\section{By Source of Infection}

When the hospital duration is split by source of infection (community-acquired or nosocomial), the changes over the years in average duration were much less pronounced (see Table 3).

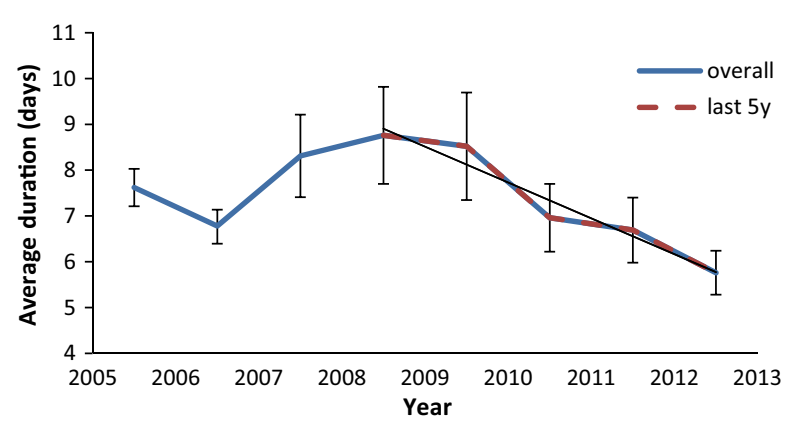

Fig. 2 Overall average duration in days for a hospital rotavirus event over time with linear time trend line for the last 5 years and standard errors of the mean
However, the average difference in duration between community-acquired (non-nosocomial) and nosocomial RV-associated hospital stay exceeded 10 days when the total hospital length of stay was considered for nosocomial infection. If only the RV-specific period of nosocomial infection was considered, the average difference was reduced to 4-5 days, with average durations of 5 and 10 days, respectively, for community-acquired and nosocomial RV-associated hospital stay (see Table 3).

\section{Age Shift}

Age had an influence on the duration of RV-induced hospital stay, especially for nosocomial infection, as shown in Fig. 4 [Kruskal-Wallis (K-W) rank test, 5 groups, K-W stats $=41.45, p<0.001]$. The average duration declined with increasing age and this trend was present with or without the vaccine introduction. In addition, we observed an absence of $\mathrm{RV}$-induced nosocomial infections in the older age groups after the vaccine introduction.

Post-vaccination (2012), the age group relatively more affected by $\mathrm{RV}$-induced nosocomial infections is the group of young infants ( $<2$ months old), who are unvaccinated $(48-24 \%=+24 \%)$ (Fig. 5) (Chi-square test for trend: $6.737 ; p<0.01)$. The relative importance increases the longer the observation period post-vaccine introduction lasts.

\section{Vaccine Impact Over Time}

Figure 6 shows the observed VI on RV-induced hospitalization over time by birth cohort and age group when compared with the pre-vaccination period. No trend for reduced VI was seen over time within each separate age group. The overall results in Fig. 6 calculate the 


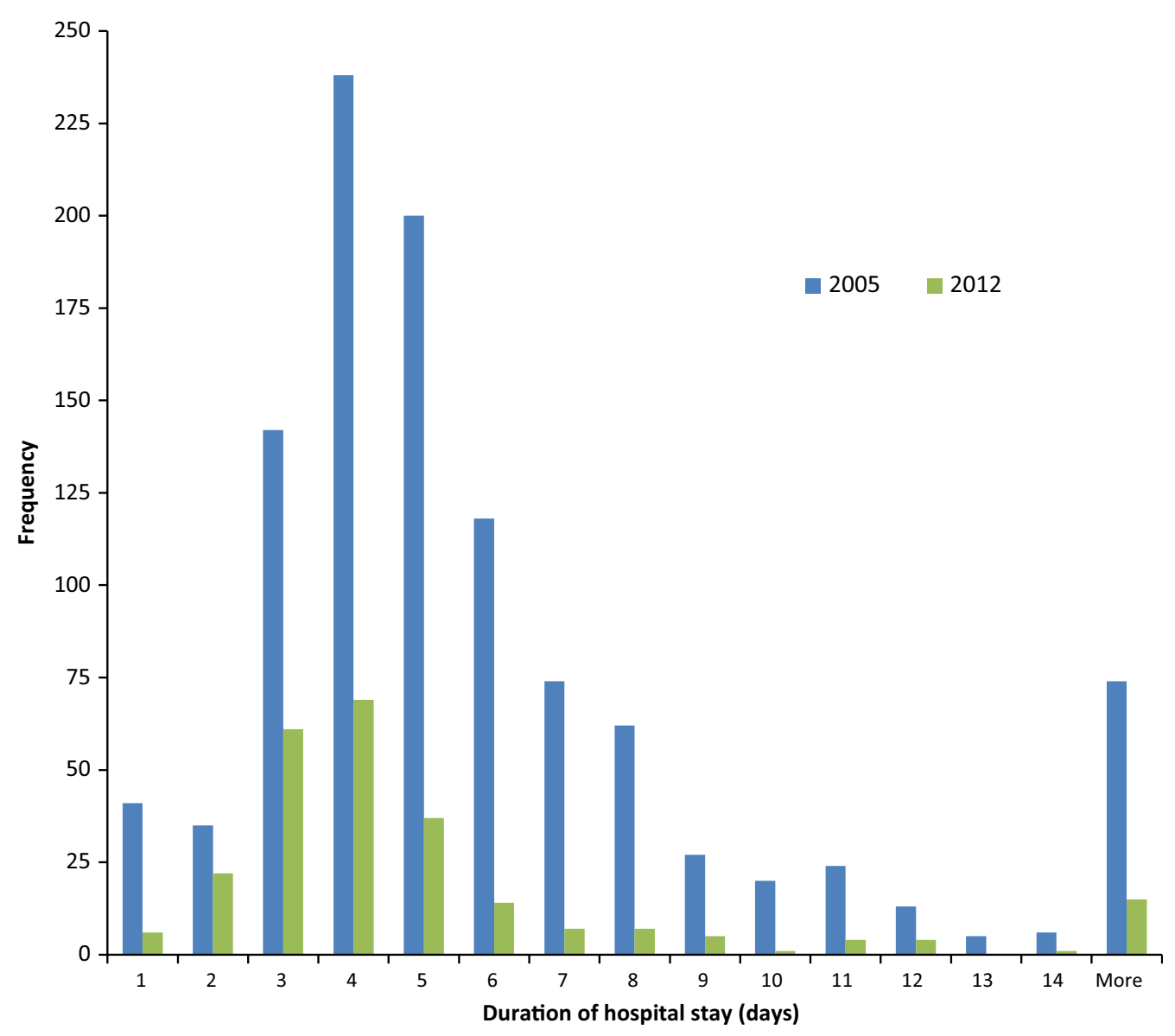

Fig. 3 Shift in the spread of duration of all rotavirus-hospital stay pre- (2005) versus post-vaccination (2012)

average VI per year, and the $95 \% \mathrm{CI}$ of the mean calculated for each year overlaps between the lowest and highest average value. However, there was a trend in the reduction of average VI across age groups: $85 \%$ in the group aged $3-12$ months; $80 \%$ in the group aged $1-2$ years; $79 \%$ in the group aged $2-3$ years; $74 \%$ in the group aged 3-4 years; and 63\% in the group aged $4-5$ years. The $95 \%$ CI calculated for each age group also overlaps between the highest and the lowest value because of the small numbers in the older age groups (95\% CI 76-91\% in the highest number of the youngest age group and $22-92 \%$ in the oldest age group). In the two oldest age groups, the denominators are small (34 and 10 cases, respectively), leading to a statistically non-significant difference between the age groups. Moreover, hospitalization events are driven by local policy in managing the disease in older children: only 2 of the 11 centers have increased hospitalization rates in the older age groups (Data on file, GSK Vaccines, Wavre, Belgium).

\section{DISCUSSION}

The benefits of RV vaccination initially reported in the literature focused on the specific mortality reduction, the decrease in hospitalizations, and the level of herd protection achieved during the first years of vaccination. These phenomena were observed 
Table 3 Average duration of hospital stay (days) by year for community-acquired RV diarrhea and RV-induced nosocomial infection

\begin{tabular}{lllllll}
\hline Year & $\begin{array}{l}\text { Number } \\
\text { of events }\end{array}$ & $\begin{array}{l}\text { Average duration } \\
\text { per event (days) }\end{array}$ & $\begin{array}{l}\text { Standard } \\
\text { deviation }\end{array}$ & Minimum & Maximum & Median \\
\hline
\end{tabular}

RV community-acquired ${ }^{a}$

$\begin{array}{lrrrrrr}2005 & 1103 & 4.87 & 4.80 & 1 & 103 & 4 \\ 2006 & 1265 & 6.03 & 11.72 & 1 & 187 & 5 \\ 2007 & 694 & 5.27 & 7.98 & 1 & 161 & 4 \\ 2008 & 462 & 5.90 & 8.53 & 1 & 134 & 4 \\ 2009 & 318 & 4.59 & 2.25 & 1 & 23 & 4 \\ 2010 & 336 & 4.26 & 2.66 & 1 & 37 & 4 \\ 2011 & 359 & 3.96 & 1.87 & 1 & 10 & 4 \\ 2012 & 294 & 4.09 & 1.90 & 1 & 16 & 4\end{array}$

$\mathrm{RV}$-induced nosocomial (full duration) ${ }^{\mathrm{b}}$

\begin{tabular}{lcccccc}
2005 & 221 & 18.28 & 25.46 & 3 & 209 & 10 \\
2006 & 134 & 12.06 & 10.52 & 3 & 73 & 9 \\
2007 & 143 & 19.92 & 48.02 & 2 & 474 & 11 \\
2008 & 69 & 17.78 & 20.30 & 4 & 125 & 10 \\
2009 & 45 & 29.60 & 44.58 & 4 & 177 & 12 \\
2010 & 50 & 20.81 & 27.81 & 4 & 162 & 10 \\
2011 & 53 & 18.71 & 24.37 & 1 & 137 & 10 \\
2012 & 33 & 16.93 & 17.36 & 4 & 101 & 12 \\
RV-induced nosocomial (rotavirus-specific period) & & & & \\
2005 & 221 & 9.21 & 17.71 & 1 & 138 & 5 \\
2006 & 134 & 5.85 & 7.95 & 1 & 64 & 4 \\
2007 & 143 & 9.73 & 26.30 & 1 & 253 & 5 \\
2008 & 69 & 9.00 & 16.67 & 1 & 120 & 4 \\
2009 & 45 & 13.66 & 23.44 & 1 & 112 & 5 \\
2010 & 50 & 10.77 & 21.89 & 1 & 136 & 4 \\
2011 & 53 & 11.20 & 19.54 & 1 & 100 & 5 \\
2012 & 33 & 8.63 & 9.44 & 1 & 45 & 5 \\
\hline$R V$
\end{tabular}

$R V$ rotavirus

a ANOVA testing, after transformation, $F(7,3631)=4.120, p=0.0002$

b ANOVA testing, after transformation, $F(7,740)=1.827, p=0.08$

c ANOVA testing, after transformation, $F(7,735)=1.108, p=0.35$ 


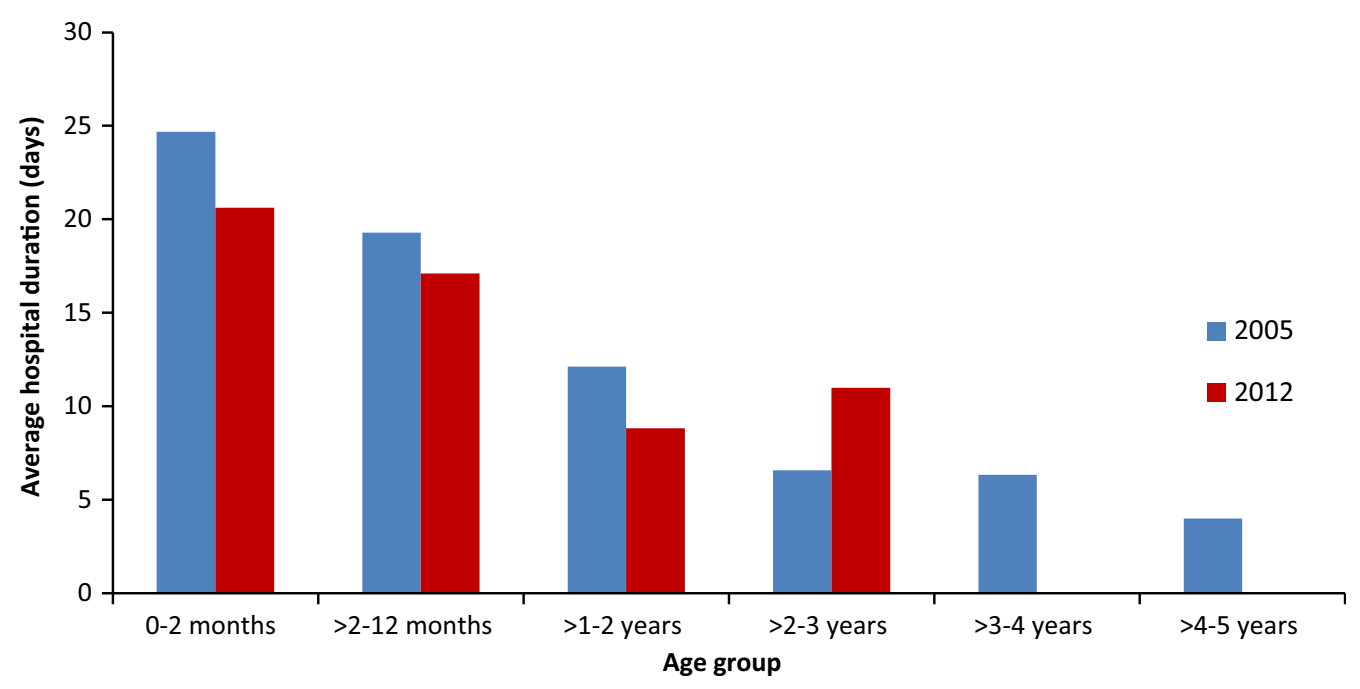

Fig. 4 Comparing the average hospital duration (days) according to age and year (2005 pre- and 2012 post-vaccination) for rotavirus-induced nosocomial infection (full duration)

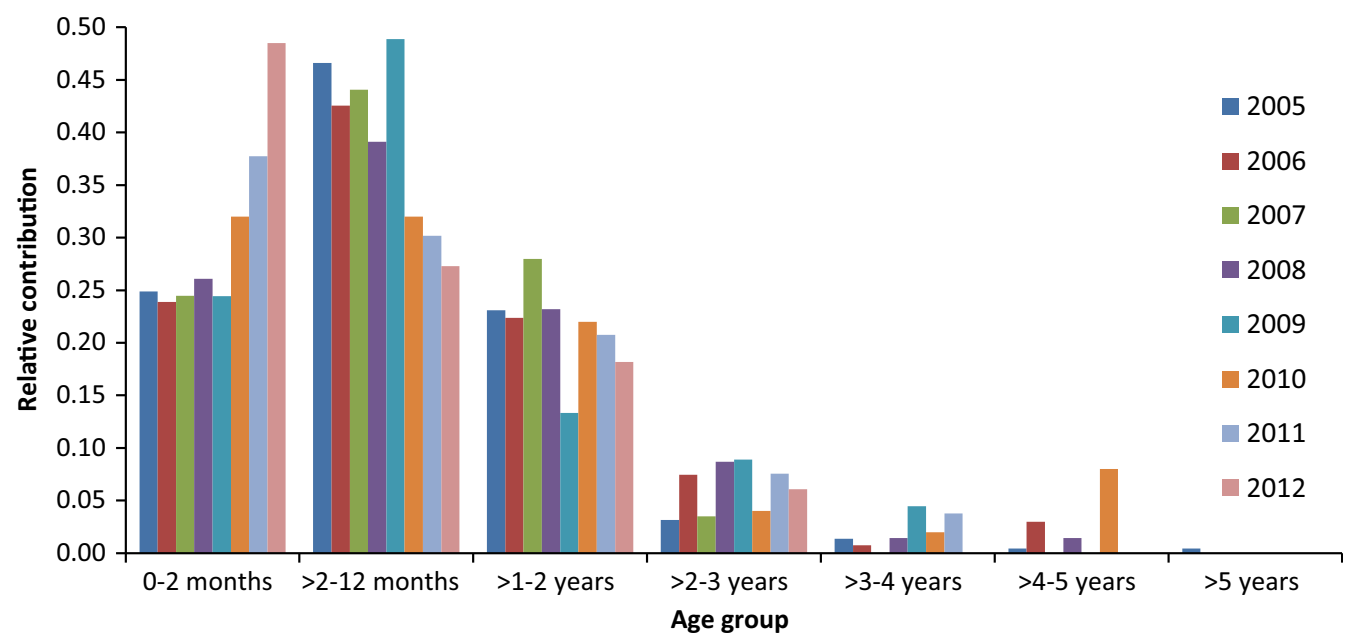

Fig. 5 The relative contribution of rotavirus-induced nosocomial infections by age group and by year

in many countries in Europe and worldwide where vaccination was implemented [19-27]. Important additional vaccine benefits that could improve QoC in the hospital environment have been less well documented and reported. It is known that RV is very contagious and that strict hygiene measures cannot always avoid the spread of the virus $[28,29]$. The present study indicates that, with the introduction of the vaccine, an important drop of $85 \%$ in nosocomial RV infections can be achieved within a few years, together with a reduction in the overall average duration of hospital stay for the disease. It is important to highlight that this overall number should be reported, as it is often the first number available in the absence of being able to differentiate between community-acquired and nosocomial infection. However, the shorter hospital stay seems to be linked to the drop in the number of 


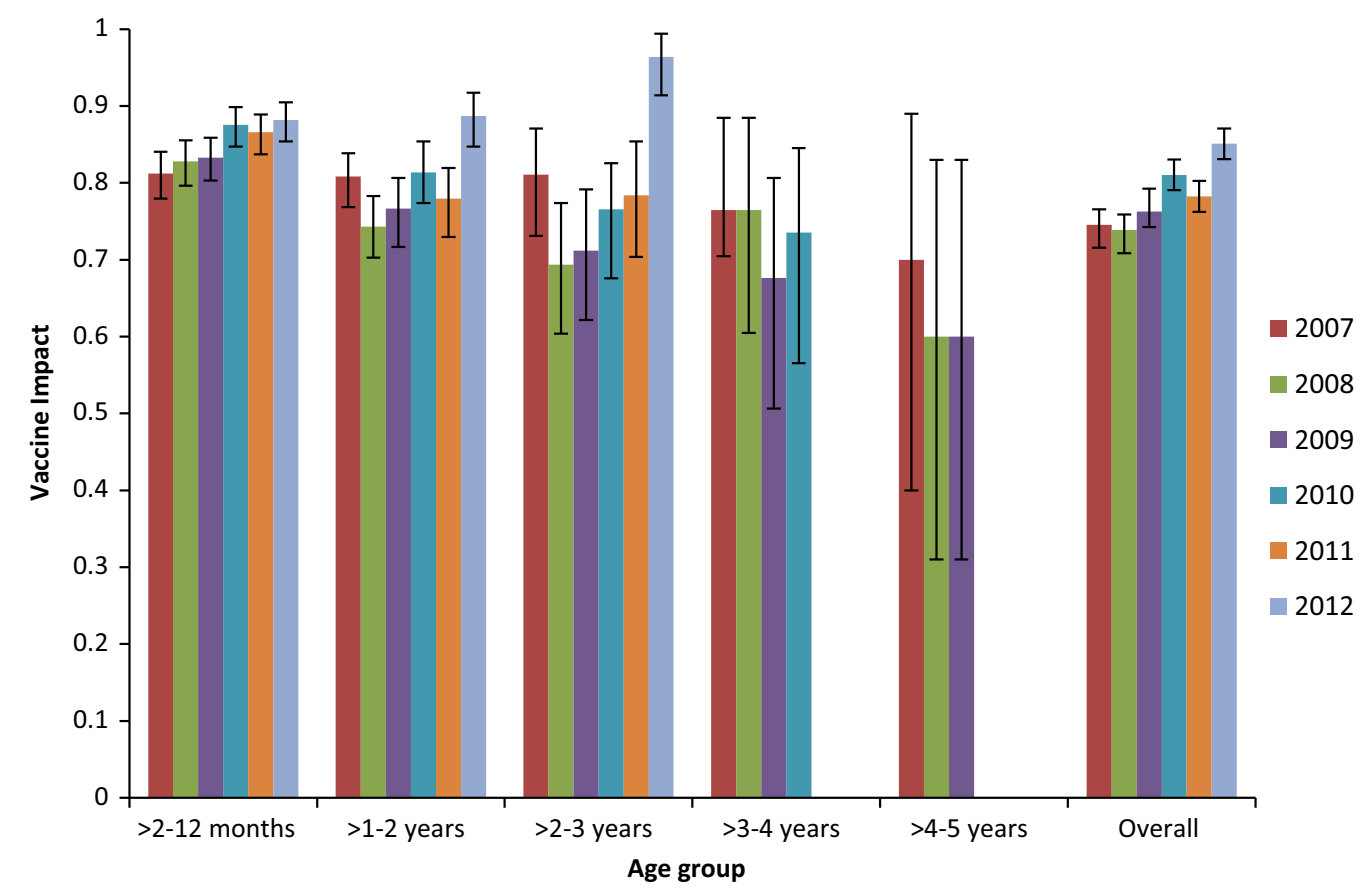

Fig. 6 Measuring rotavirus vaccine impact by year, birth cohort, and age group

nosocomial infections, which on average have a much longer duration than the community-acquired infections, rather than to a shorter hospital stay specific for community-acquired RV diarrhea and/or $\mathrm{RV}$-induced nosocomial infection. The drop in nosocomial infections and the shorter hospital stay will benefit young patients who are admitted to the hospital for reasons other than diarrhea, as they will now be exposed to a much lower risk of RV infection after the introduction of the vaccine. This will benefit their recovery because the shorter duration of hospital stay will reduce their risk of exposure to any other nosocomial infection source in the hospital environment [16].

Figure 5 shows that after vaccine introduction the distribution of $\mathrm{RV}$ hospitalization for nosocomial infections shifted towards the very young age group where infants are still unvaccinated. This process increased progressively over the successive years of the vaccination program (see the youngest age group in Fig. 5). We, therefore, evaluated the data on duration of hospital stay to investigate whether more severe cases were hospitalized in more recent years post-vaccination among infants, when compared with the pre-vaccination period in the same age group. Figure 7 shows the results in the group aged $<2$ months for RV-induced nosocomial infection and RV-induced community-acquired diarrhea, comparing the year 2005 (pre-vaccination) with the year 2012 (post-vaccination, the latest year of the observation). There was little difference between 2005 and 2012 in the duration of hospital stay for RV-induced community-acquired diarrhea events (the lines are close together at the left side of the graph). In contrast, among RV-induced nosocomial infections there was a substantial reduction in 


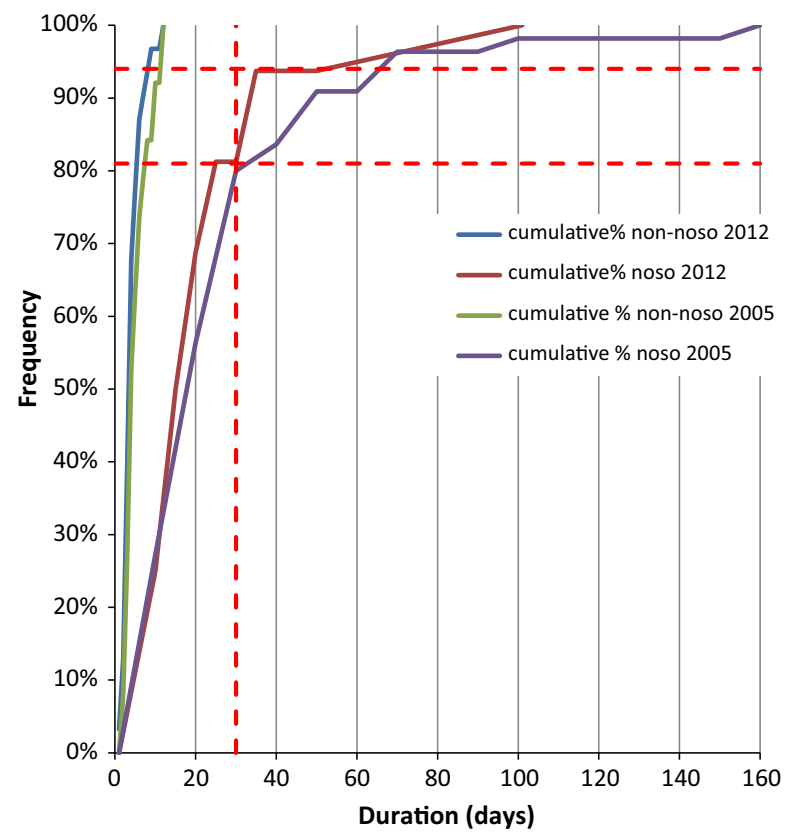

Fig. 7 Comparison of the cumulative frequency (\%) of patients as a function of the duration of hospital stay for rotavirus-induced nosocomial and community-acquired infection of infants $<2$ months old for the year 2005 and the year 2012. Red dashed lines indicate the frequency of hospitalizations $>30$ days in 2005 and 2012

the frequency of patients with long hospital stays ( $>30$ days of hospitalization) after the vaccine was introduced. Figure 7 also shows the frequency of hospitalizations $>30$ days in 2005 and 2012, and indicates a reduction of $14 \%$ in these long hospitalizations in the post-vaccine period (2012) compared with the pre-vaccine period (2005). It suggests a trend to less severe disease events in very young infants after the vaccine was introduced. The rank test (Mann-Whitney $U$ test) did not report a statistically significant difference in duration between the two periods, although the median was 4 days lower for the 2012 data (median: 18 days in 2005 and 14 days in 2012; $p=0.45$ ).

This analysis raises two questions. First, whether it is rational to include long hospital stays in the analysis of nosocomial infections, as those long stays are probably not caused by the rotavirus infections but more by the primary cause of hospitalization. The graph may, therefore, help to identify a reasonable cutoff for hospitalization lengths that still contribute to the average duration of the rotavirus nosocomial infection. In Fig. 7, a cutoff of 25-30 days seems reasonable, although the results of the cutoff could be different in different environments. Second, whether there is a need for earlier vaccination, closer to the day of birth, as it is mainly in very young infants that additional benefit of the vaccine can be expected and where the current disease burden is particularly concentrated. Further research is required [30-32].

The question of whether the VI is maintained over time needs to be answered cautiously. There are two time dimensions to be considered in the analysis. One is a horizontal timeline with annual evaluations of the impact, and the other is a diagonal timeline as individuals move to the next year (horizontal shift) and at the same time grow older (vertical shift). This is called a cohort analysis versus a population analysis [33]. When the results are compared horizontally, good consistency is observed over time within each age group as reported in Fig. 6. The overall results for each annual birth cohort indicate a slight increase in average VI, but that is due to the last two birth cohorts where data were not available for all the age groups as the individuals in these birth cohorts have not yet attained the older ages.

Considered from a cohort point of view, a trend of VI decrease was observed by age group (also reported in Fig. 6). This could be related to a real decrease in VI, a change in management of events among the older children, or a change in testing leading to fewer cases detected and reported in the older age groups. Essentially, two centers out of the eleven had a policy to keep older children in the hospital. Therefore, 
the rules of disease management across ages and centers should be maintained to measure a real decline in VI, rather than some possible change in disease or diagnostic management because of particular pressures.

There are weaknesses in the RotaBIS study, because it has a retrospective design without full control of the denominator. In addition, no rules have been imposed on precisely how to manage children for hospitalization independent of age. It could, therefore, be that the data here observed are not only a consequence of the vaccination impact but could also be a result of additional measures taken in the hospital environment to improve control of infectious diseases during recent years. This has consequences when handling small numbers and reporting relative values, such as the VI in the age group above 3 years. However, the highest disease burden is in the group aged $<2$ years, and we observed quite consistent results over time in this group [34]. Finally, information on the vaccination status of the children when they are hospitalized is missing from the study. We are working on ways to collect that information in subsequent runs of the RotaBIS study in the coming years, as it is important to know how many of the hospitalized children were exposed to the vaccine. However, the attractiveness of the RotaBIS study is the low burden in collecting the data, the simple analyses performed and communicated, which sometimes raise additional new research questions, and the opportunity to obtain real-world results that can be compared with model output predictions (for instance, related to herd protection). It gives valuable information on the disease and whether the vaccine maintains its impact over time, which will be useful to decision-makers evaluating $\mathrm{RV}$ vaccination programs.

\section{CONCLUSION}

The RotaBIS study illustrates additional results that $\mathrm{RV}$ vaccination may generate within a hospital environment, besides reducing the number of hospitalizations. The new results focus on the reduction in nosocomial RV infections $(-85 \%)$, leading to a shorter duration in hospital stay (on average $<2$ days per RV event). Consequently, better QoC in the hospital environment can be developed, enhancing better health care delivery because of the reduction in bed occupancy during the peak period of rotavirus disease. From the data analyzed over a 6-year period, the VI was maintained over time in the younger age groups. We observed a slight decrease in the older age groups above 3 years, which could be better explained by changes in local disease and diagnostic management rules than by a real decline in VI.

\section{ACKNOWLEDGMENTS}

Rotarix $^{\mathrm{TM}}$ is a trademark of the GSK group of companies. GlaxoSmithKline Biologicals SA (Rixensart, Belgium) was the funding source for all stages of the study conduct and analysis (NCT01563146) and for all costs associated with the development and the publishing of the present manuscript. All named authors meet the International Committee of Medical Journal Editors (ICMJE) criteria for authorship for this manuscript, take responsibility for the integrity of the work as a whole, and have given final approval to the version to be published. All authors had full access to the data and the corresponding author had final responsibility to submit for publication. The authors would like to thank the investigators of The Rotavirus Belgium Impact Study (RotaBIS study) group who participated in this study: Marc Verghote, Centre Hospitalier Régional de Namur, 
Namur; Eddy Bodart, Cliniques Universitaires, Université catholique de Louvain (UCL) de Mont Godinne, Yvoir; Stefaan Van Eldere, Imeldaziekenhuis, Bonheiden; Patricia Vandecandelaere, Regionaal Ziekenhuis Jan Yperman, O.L.Vrouw, Ieper; Peggy Bruynseels, Ziekenhuis Netwerk Antwerpen, Antwerpen; Jose Ramet, Universitair Ziekenhuis Antwerpen, Edegem; Hajer Lassoued, Clinique Notre Dame, Charleroi. The authors would like to thank Business \& Decision Life Sciences platform for editorial assistance and manuscript coordination, on behalf of GSK Vaccines. Sarah Fico coordinated manuscript development, editorial support and provided medical writing support. The authors would also like to thank Carole Nadin (Fleetwith Ltd, on behalf of GSK Vaccines) for reviewing the manuscript for English usage.

Disclosures. B. Standaert is an employee of the GSK group of companies and holds stock options in the GSK group of companies. $\mathrm{X}$. Li is an employee of the GSK group of companies. N. Schecroun reports consulting fees to her institution, Keyrus Biopharma, and travel to congress and accommodation from the GSK group of companies. D. Strens' institution received consulting fees from the GSK group of companies for work under consideration for publication and outside the submitted work. M. Raes' institution received a consulting fee or honorarium from the GSK group of companies for the submitted work. Outside the submitted work, M. Raes received funding from the GSK group of companies for board membership and lectures and has received funding for travel, accommodation and meetings expenses.

Compliance with Ethics Guidelines. The data were anonymized before any analysis occurred. All procedures followed were in accordance with the ethical standards of the responsible committee in each center. Approval for the study was obtained from each center each year the data were collected.

Open Access. This article is distributed under the terms of the Creative Commons Attribution-NonCommercial 4.0 International License (http://creativecommons.org/licenses/ by-nc/4.0/), which permits any noncommercial use, distribution, and reproduction in any medium, provided you give appropriate credit to the original author(s) and the source, provide a link to the Creative Commons license, and indicate if changes were made.

\section{REFERENCES}

1. Parashar UD, Gibson CJ, Bresee JS, Glass RI. Rotavirus and severe childhood diarrhea. Emerg Infect Dis. 2006;12(2):304-6.

2. Forster J, Guarino A, Parez N, et al. Hospital-based surveillance to estimate the burden of rotavirus gastroenteritis among European children younger than 5 years of age. Pediatrics. 2009;123(3):e393-400.

3. Hartwig S, Uhari M, Renko M, et al. Hospital bed occupancy for rotavirus and all cause acute gastroenteritis in two Finnish hospitals before and after the implementation of the national rotavirus vaccination program with RotaTeq(R). BMC Health Serv Res. 2014;14:632.

4. Mullins JA, Lamonte AC, Bresee JS, Anderson LJ. Substantial variability in community respiratory syncytial virus season timing. Pediatr Infect Dis J. 2003;22(10):857-63.

5. Chandran A, Fitzwater S, Zhen A, Santosham M. Prevention of rotavirus gastroenteritis in infants and children: rotavirus vaccine safety, efficacy, and potential impact of vaccines. Biologics. 2010;4:213-29.

6. Ruiz-Palacios GM, Pérez-Schael I, Velazquez FR, et al. Safety and efficacy of an attenuated vaccine against severe rotavirus gastroenteritis. N Engl J Med. 2006;354(1):11-22.

7. Vesikari T, Karvonen A, Prymula R, et al. Efficacy of human rotavirus vaccine against rotavirus gastroenteritis during the first 2 years of life in 
European infants: randomised, double-blind controlled study. Lancet. 2007;370(9601):1757-63.

8. Vesikari T, Matson DO, Dennehy P, et al. Safety and efficacy of a pentavalent human-bovine (WC3) reassortant rotavirus vaccine. $\mathrm{N}$ Engl $\mathrm{J}$ Med. 2006;354(1):23-33.

9. Raes M, Strens D, Vergison A, Verghote M, Standaert B. Reduction in pediatric rotavirus-related hospitalizations after universal rotavirus vaccination in Belgium. Pediatr Infect Dis J. 2011;30(7):e120-5.

10. Braeckman T, Van HK, Raes $M$, et al. Rotavirus vaccines in Belgium: policy and impact. Pediatr Infect Dis J. 2011;30(1 Suppl):S21-4.

11. World Health Organization. WHO and UNICEF estimates of immunization coverage: 2015 revision. http://www.who.int/immunization/monitoring _ surveillance/data/bel.pdf. Accessed 29 June 2016.

12. Standaert B, Strens D, Alwan A, Raes M. Medium- to long-term impact of rotavirus vaccination on hospital care in Belgium: a 7-year follow-up of the Rotavirus Belgium Impact Study (RotaBIS). Infect Dis Ther. 2016;5(1):31-44.

13. Bruijning-Verhagen $\mathrm{P}$, Quach C, Bonten $\mathrm{M}$. Nosocomial rotavirus infections: a meta-analysis. Pediatrics. 2012;129(4):e1011-9.

14. Gundeslioglu OO, Tekin R, Cevik S, Palanci Y, Yazicioglu A. The effects of nosocomial rotavirus gastroenteritis on the length of hospital stay and cost. J Infect Dev Ctries. 2016;10(2):163-7.

15. Pitzer VE, Atkins KE, de Blasio BF, et al. Direct and indirect effects of rotavirus vaccination: comparing predictions from transmission dynamic models. PLoS One. 2012;7(8):e42320.

16. Standaert B, Alwan A, Strens D, Raes M, Postma MJ. Improvement in hospital quality of care (QoC) after the introduction of rotavirus vaccination: an evaluation study in Belgium. Hum Vaccin Immunother. 2015;11(9):2266-73.

17. Hanquet G, Ducoffre G, Vergison A, et al. Impact of rotavirus vaccination on laboratory confirmed cases in Belgium. Vaccine. 2011;29(29-30):4698-703.

18. Wilson E. Probable inference, the law of succession and statistical inference. J Am Stat Assoc. 1927;22:209-12.

19. Patel MM, Steele D, Gentsch JR, et al. Real-world impact of rotavirus vaccination. Pediatr Infect Dis J. 2011;30(1 Suppl):S1-5.

20. Linhares AC, Justino MC. Rotavirus vaccination in Brazil: effectiveness and health impact seven years post-introduction. Expert Rev Vaccines. 2014;13(1):43-57.

21. Richardson V, Hernandez-Pichardo J, Quintanar-Solares $\mathrm{M}$, et al. Effect of rotavirus vaccination on death from childhood diarrhea in Mexico. N Engl J Med. 2010;362(4):299-305.

22. Atchison CJ, Stowe J, Andrews N, et al. Rapid Declines in Age Group-Specific Rotavirus Infection and Acute Gastroenteritis Among Vaccinated and Unvaccinated Individuals Within 1 Year of Rotavirus Vaccine Introduction in England and Wales. J Infect Dis. 2016;213(2):243-9.

23. Paulke-Korinek M, Kollaritsch H, Aberle SW, et al. Sustained low hospitalization rates after four years of rotavirus mass vaccination in Austria. Vaccine. 2013;31(24):2686-91.

24. Vesikari T, Sutherland D, Jackson AE. Report of the 'European Expert Meeting on Rotavirus Vaccination', Tampere, Finland, 19-20 May 2009. Vaccine. 2009;27(52):7222-7.

25. Hungerford D, Read JM, Cooke RP, et al. Early impact of rotavirus vaccination in a large paediatric hospital in the UK. J Hosp Infect. 2015;93(2):117-20.

26. Standaert B, Gomez JA, Raes M, et al. Impact of rotavirus vaccination on hospitalisations in Belgium: comparing model predictions with observed data. PLoS ONE. 2013;8(1):e53864.

27. Payne DC, Selvarangan R, Azimi PH, et al. Long-term consistency in rotavirus vaccine protection: RV5 and RV1 vaccine effectiveness in US children, 2012-2013. Clin Infect Dis. 2015;61(12):1792-9.

28. Waisbourd-Zinman O, Ben-Ziony S, Solter E, et al. Hospitalizations for nosocomial rotavirus gastroenteritis in a tertiary pediatric center: a 4-year prospective study. Am J Infect Control. 2009;37(6):465-9.

29. Gleizes O, Desselberger U, Tatochenko V, et al. Nosocomial rotavirus infection in European countries: a review of the epidemiology, severity and economic burden of hospital-acquired rotavirus disease. Pediatr Infect Dis J. 2006;25(1 Suppl):S12-21.

30. Bines JE, Danchin M, Jackson P, et al. Safety and immunogenicity of RV3-BB human neonatal rotavirus vaccine administered at birth or in infancy: a randomised, double-blind, placebo-controlled trial. Lancet Infect Dis. 2015;15(12):1389-97.

31. Vesikari T. Neonatal rotavirus vaccination making headway. Lancet Infect Dis. 2015;15(12):1362-3. 
32. Vesikari T, Van DP, Giaquinto C, et al. European Society for Paediatric Infectious Diseases consensus recommendations for rotavirus vaccination in Europe: update 2014. Pediatr Infect Dis J. 2015;34(6):635-43.

33. Ethgen O, Standaert B. Population- versus cohort-based modelling approaches. Pharmacoeconomics. 2012;30(3):171-81.
34. Bruun T, Salamanca BV, Bekkevold T, et al. Burden of rotavirus disease in Norway: using national registries for public health research. Pediatr Infect Dis J. 2016;35(4):396-400. 